Comparative Biochemistry and Physiology - Part A: Molecular \& Integrative Physiology

Volume 128, Issue 2, February 2001, Pages 251-257

\title{
Arachidonic and eicosapentaenoic acids in tissues of the firefly, Photinus pyralis (Insecta: Coleoptera)
}

A.R. Nor Aliza, Jon C. Bedick, Rico L. Rana, Hasan Tunaz, W.Wyatt Hoback, David W. Stanley

\begin{abstract}
We report on the presence of high proportions of arachidonic acid (20:4n-6) and eicosapentaenoic acid (20:5n-3) in the tissue lipids of adult fireflies, Photinus pyralis. Arachidonic acid typically occurs in very small proportions in phospholipids (PLs) of terrestrial insects, ranging from no more than traces to less than $1 \%$ of PL fatty acids, while 20:5n-3 is often missing entirely from insect lipids. Contrarily, 20:4n-6 made up approximately $21 \%$ of the PL fatty acids prepared from whole males and females, and from heads and thoraces prepared from males. Proportions of 20:4n-6 associated with PLs varied among tissues, including approximately $8 \%$ for male gut epithelia, $13 \%$ for testes, and approximately $25 \%$ for light organs and body fat from males. Substantial proportions of 20:5n-3 were also associated with PLs prepared from male firefly tissues, including $5 \%$ for body fat and $8 \%$ for light organs. Because 20:4n-6 and 20:5n-3 are precursors for biosynthesis of prostaglandins and other eicosanoids, we considered the possibility that firefly tissues might produce eicosanoids at exceptionally high rates. Preliminary experiments indicated otherwise. Hence, fireflies are peculiar among terrestrial insects with respect to maintaining high proportions of PL 20:4n-6 and 20:5n-3.
\end{abstract}

Keywords: Firefly; Eicosapentaenoic acid; Arachidonic acid; Photinus pyralis 